\title{
A beamline for attosecond UV pump - XUV probe experiments
}

\author{
Mara Galli ${ }^{1,2^{*}}$, Vincent Wanie ${ }^{1,3}$, Erik Peter Månsson ${ }^{4}$, Andrea Trabattoni ${ }^{4}$, Francois \\ Légaré $^{3}$, Fabio Frassetto ${ }^{5}$, Luca Poletto ${ }^{5}$, Mauro Nisoli ${ }^{1,2}$, and Francesca Calegari ${ }^{4,6,1}$ \\ ${ }^{1}$ Institute of Photonics and Nanotechnologies-Consiglio Nazionale delle Ricerche, Piazza Leonardo \\ da Vinci 32, 20133 Milano, Italy \\ ${ }^{2}$ Department of Physics, Politecnico di Milano, Piazza Leonardo da Vinci 32, 20133 Milano, Italy \\ ${ }^{3}$ Institut National de la Recherche Scientifique, Centre Énergie Matériaux et Télécommunications, \\ 1650 Blvd. Lionel-Boulet, Varennes, J3X1S2, Canada \\ ${ }^{4}$ Center for Free-Electron Laser Science, DESY, Notkestr. 85, 22607 Hamburg, Germany \\ ${ }^{5}$ Institute for Photonics and Nanotechnologies CNR-IFN, Via Trasea 7, 35131 Padova, Italy \\ ${ }^{6}$ Department of Physics, University of Hamburg, Luruper Chaussee 149, D-22761 Hamburg, \\ Germany
}

\begin{abstract}
A time resolved setup combining sub-2fs UV pump pulses with attosecond XUV probe pulses is presented. This scheme will allow the UV photo-induced electron dynamics in bio-chemically relevant molecules to be investigated with unprecedented time resolution.
\end{abstract}

\section{Introduction}

Upon photo-exposure to ultraviolet (UV) light, the building blocks of biomolecules become electronically excited. In DNA this process may lead to mutagenic miscoding of the sequence and irreparable damage [1]. It has been proven that UV-light driven DNA damage finds its origin in ultrafast molecular processes occurring on a time scale below 1ps [2].

In this contest, the role of the UV activated electron dynamics in the photochemistry of this complex molecule is still not known, indeed the lack of a technology for the generation of few-fs UV pulses has limited the investigation of the UV photo-induced processes in bio-chemically relevant molecules to the time scale above tens of femtoseconds, mostly addressing the nuclear dynamics [3].

Recently, attosecond technology has been proven to be a valuable tool to study electron dynamics in biomolecules with few-fs time resolution [4,5] In the conventional scheme for attosecond time resolved experiments, extreme ultraviolet (XUV) attosecond pump pulses are typically combined with few-fs near infrared (NIR) probe pulses. This approach however implies the use of XUV ionizing radiation in the first step, preventing the study of neutral species, and the use NIR pulses in the probing step often inducing multi-photon processes.

Here we propose a novel approach based on the combination of sub-2fs UV pulses, meant to trigger the electron dynamics in biorelevant molecules, with isolated XUV attosecond pulses in order to probe the UV photo-induced processes with sub-fs time 
resolution. The UV pulses are generated via frequency up-conversion from few-fs NIR pulses in a high-pressure gas cell. This generation method combined with propagation in vacuum, allows for limiting the dispersive effects. The ultrashort UV pulses are noncollinearly combined with XUV attosecond pulses produced with the polarization gating approach [6].

\section{Results}

The attosecond beamline is schematically represented in Fig. 1. The beamline is seeded by $5 \mathrm{fs}$ NIR pulses with $2 \mathrm{~mJ}$ energy, $1 \mathrm{kHz}$ repetition rate and a controlled carrier-envelope phase (CEP) with a residual error of $200 \mathrm{mrad}$ [7].

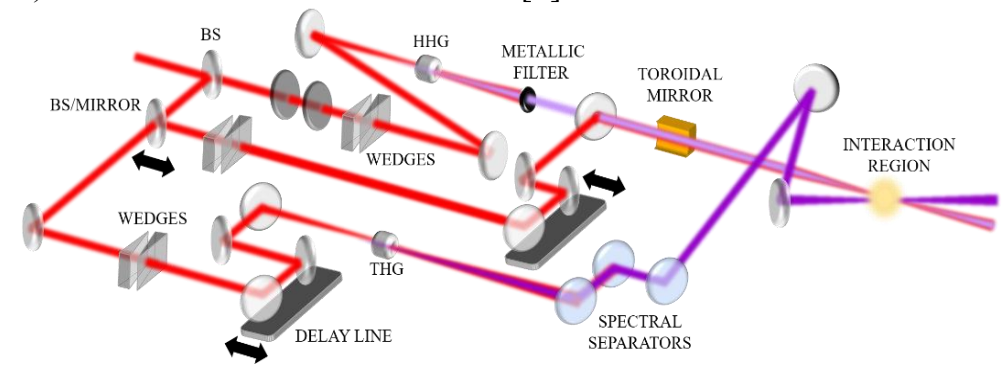

Fig. 1 Scheme of the attosecond beamline

$70 \%$ of the incoming radiation is focused in a pulsed gas cell for the generation of XUV radiation via the high-order harmonic generation (HHG) process. By exploiting the polarization gating technique isolated pulses can be obtained with a cut-off energy around 30-40 eV and a time duration of approximately 200as (Fig. 2a). A 150-nm thick aluminium filter is placed after the XUV generation cell in order to filter out the residual NIR light, transmitting radiation above $17 \mathrm{eV}$.

The remaining $30 \%$ of the NIR incoming radiation can be either used in the conventional XUV-pump NIR-probe approach for the temporal characterization of the XUV attosecond pulses [8], or it can be used to generate UV radiation. Third harmonic generation (THG) of 5fs, 250-uJ NIR pulses in a non-dispersive medium is used to produce UV pulses with 150nJ energy and a bandwidth ranging between $210 \mathrm{~nm}$ and $320 \mathrm{~nm}$. The generated UV spectrum supports a transform limited temporal duration of 0.8 fs FWHM (Fig. $2 b-2 c$ ), thus potentially indicating the possibility to generate deep ultraviolet pulses in the attosecond range.
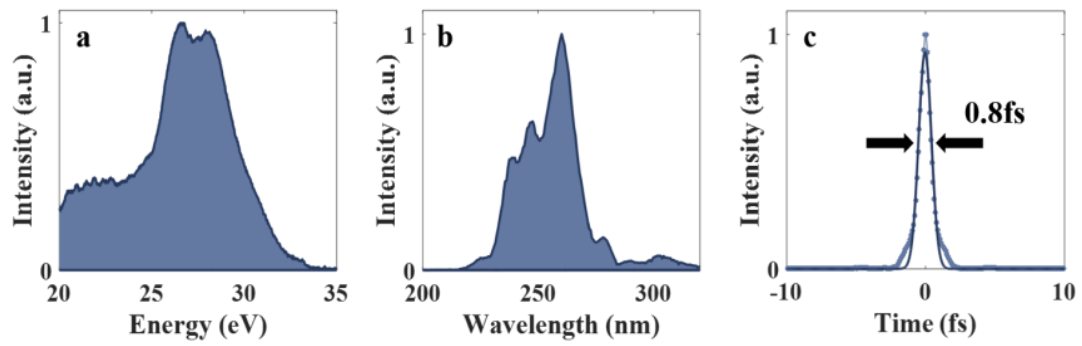

Fig. 2 (a) Typical spectrum of the isolated XUV attosecond pulses, (b) typical spectrum of the UV pulses and (c) transform limited temporal profile of the UV pulses fitted with a Gaussian (1.1 fs FWHM) (c). 
UV generation has been achieved in a high-pressure fused-silica gas cell properly designed and realized with the FLICE technique [9]. A small chamber with three differential pumping stages has been implemented, in order to limit the THG process to a few millimetres along the Rayleigh range and, at the same time, to preserve the high vacuum required in the interaction region to perform the experiments. The gas used for THG is recirculated using a combination of a sealed pump and a compressor to limit the gas consumption both in the case of generation in Argon (1.5 bar) or in Neon (up to 7 bars). In order to suppress the generating NIR radiation, three dielectric wedged dielectric beam separators have been added in the optical path after the THG cell. After the beam separators, the residual NIR pulse energy is $28 \mathrm{~nJ}$, which is considered to be negligible on target.

XUV pules can be recombined with NIR pulses, for attosecond pulse characterization, using a collinear geometry based on a drilled mirror. In this case, both beams are focused in the interaction region by a gold-coated toroidal mirror. On the other hand, a non-collinear geometry is adopted to combine the XUV pulses with the UV pulses. This approach allows to preserve the whole energy of the UV arm. The relatively small angle $(6.6 \mathrm{mrad})$ allows to preserve a sufficiently high temporal resolution for the experiment, which is only limited by the UV pulse duration. The beamline is also provided with an XUV spectrometer to monitor in real time the generation of the attosecond pulses during the experiments: a new design has been adopted to give more compactness to the instrument.

Temporal characterization of the UV pulses has been achieved via cross-correlation with the NIR field. Resonance enhanced multiphoton ionization from the $6 p$ Rydberg series of Xenon [10] was exploited to retrieve a $2 \mathrm{fs}$ UV pulse duration.

\section{Conclusions}

We presented a new versatile attosecond beamline to perform UV-pump XUV-probe experiments on bio-chemically relevant molecules with unprecedented time resolution. This new scheme will allow to finally disclose the role of the electron dynamics in the UV photochemistry of complex molecules of biological interest.

\section{References}

1. E. Crespo-Hernandez, B. Cohen, P. M. Hare, B. Kohler, Chem.Rev.104, 1977 (2004)

2. T. Gustavsson, R. Improta and D. Markovitsi, J. Chem. Phys. Lett 1, 2025-2030, (2010)

3. H. Satzger, D. Townsend, M. Z. Zgierski, S. Patchkovskii, S. Ullrich, A. Stolow, PNAS $103,10196(2006)$

4. F. Calegari, D. Ayuso, A. Trabattoni, L. Belshaw, S. De Camillis, S. Anumula, F. Frassetto, L. Poletto, A. Palacios, P. Decleva, J. Greenwood, F. Martin, M. Nisoli, Science 346 (6207) 336-339, (2014)

5. M. Nisoli, P. Decleva, F. Calegari, A. Palacios, F. Martín, Chem. Rev. 117 (16), 1076010825 (2017)

6. G. Sansone, E. Benedetti, F. Calegari, C. Vozzi, L. Avaldi, R. Flammini, L. Poletto, P. Villoresi, C. Altucci, R. Velotta, S. Stagira, S. De Silvestri, M. Nisoli, Science 314, 443 (2006)

7. M. Nisoli, S. De Silvestri and O. Svelto, Appl. Phys. Lett. 68, 2793 (1996)

8. J. Itatani, F. Quéré, G. L. Yudin, M. Yu. Ivanov, F. Krausz and P.B. Corkum, Phys. Rev. Lett. 88, 173903 (2002)

9. R. R. Gattas, E. Mazur, Nat. Phot. 2, 219-225 (2008)

10. L. Allen, D. G. C. Jones, D. G. Schofield, J. Opt. Soc. Am. 59,7 (1969) 